\title{
Patient Preferences in the Treatment of Vaginal Candidiasis
}

\author{
Mark G. Martens \\ Obstetrics and Gynecology, Reading Hospital, West Reading, PA, USA \\ Email: mark.martens@towerhealth.org
}

How to cite this paper: Martens, M.G. (2018) Patient Preferences in the Treatment of Vaginal Candidiasis. Open Journal of Obstetrics and Gynecology, 8, 1147-1155. https://doi.org/10.4236/ojog.2018.812116

Received: August 24, 2018

Accepted: October 12, 2018

Published: October 15, 2018

Copyright (C) 2018 by author and Scientific Research Publishing Inc. This work is licensed under the Creative Commons Attribution International License (CC BY 4.0).

http://creativecommons.org/licenses/by/4.0/

\begin{abstract}
Vulvovaginal candidiasis (VVC) is one of the most frequent problems facing women and their healthcare professionals (HCP). It is difficult for providers to understand the prevalence of VVC because effective over the counter treatments (OTC), are available. It is expected that there are a great many more episodes of VVC in our patient population, as the frequency of self-treatment, success and satisfaction for those that use OTC products prior to seeing a women's health care provider is unknown. In this study; healthcare providers were given OTC miconazole/tioconazole units for free distribution to patients for whom they diagnosed VVC by exam in their offices. Surveys for both HCP and patients were also distributed to determine the initial or recurrent nature of their symptoms and their satisfaction with their treatments. 1265 OBGYNs and $1821 \mathrm{NP} / \mathrm{CNM} / \mathrm{PAs}$ reported on over 19,000 patients receiving a single complete treatment. Among HCPs, treatment efficacy was the primary reason for recommending miconazole/tioconazole. However, rapid onset of symptom relief, safety vs. fluconazole, patient preference for the less messy ovule, avoidance with drugs metabolized by the liver, and resistance to, or failure on, fluconazole were additional reasons noted for topical preference. Overall, patient satisfaction $(n=317)$ was high at $95 \%$, with $97 \%$ of patients reporting that they would use miconazole again. Importantly, $100 \%$ of first time sufferers $(\mathrm{n}=95)$ would use miconazole again. The few patients dissatisfied with miconazole (2\%), reported no symptom relief, burning after use, an "allergic reaction", pain from the applicator or a messy discharge. Most patients using the external symptom relief products were satisfied with the external cream (93\%) and wipes (96\%). Almost all patients reported their yeast infection was cured (91\%) and that they experienced quick symptom relief (94\%), despite the prevalence of recurrent vs first time VVA sufferers that presented to offices in this trial. Most patients were cured and satisfied with their treatment, therefore supporting the use of topical miconazole/tioconazole as first line therapy for vulvovaginal candidiasis patients
\end{abstract}


presenting to a healthcare office.

\section{Keywords}

Candidiasis, Miconazole, Patient Preference, Vulvovaginal Yeast Infections

\section{Introduction}

The most common cause of vulvovaginal candidiasis (VVC) is Candida albicans but other candidal species or yeasts may be responsible for causing the clinical syndrome [1].

Typical symptoms of VVC [2] include pruritus, vaginal soreness, dyspareunia, dysuria, and abnormal vaginal discharge. Approximately $75 \%$ of women will have at least one episode of VVC during their lifetime, and between $40 \%$ and $45 \%$ will have two or more episodes. Based on clinical presentation, microbiology, host factors, and response to therapy, VVC can be classified as either uncomplicated or complicated. Up to $20 \%$ of women will have complicated VVC, requiring special consideration regarding diagnosis and treatment [3].

For many patients, a short-course of a topical formulation, either as a single dose or a 3-day regimen, can be used to treat uncomplicated VVC effectively. According to the CDC, the topically applied azole drugs are more effective than nystatin. Treatment with azoles results in relief of symptoms and negative cultures in $80 \%-90 \%$ of patients who complete therapy.

Topical agents usually cause no systemic side effects, although local burning or irritation might occur. Healthcare professionals should keep in mind that clinically important interactions can occur when oral azoles agents are administered with other drugs [4] [5] [6].

One of the topical azoles, miconazole, has been shown to be just as effective at curing VVC as fluconazole, the leading prescription pill. In a clinical study that compared 7-day topical miconazole to prescription fluconazole, there was no statistically significant difference in clinical cure rates at 14 days post-treatment.

Topical agents, such as miconazole begin to work on contact, curing VVC just as effectively as fluconazole while relieving symptoms much sooner, is effective for both albicans and non-albicans species of yeast, and treats a broader spectrum of VVC than prescription fluconazole [7] [8] [9] [10].

For non-albicans VVC, a non-fluconazole azole is recommended by CDC, for a duration of 7 - 14 days. Practically, if patients use a 1-day, 3-day or 7-day course of a topical azole and they are still symptomatic after a week, following up with a second course of intra-vaginal therapy for 7 days is advised.

In 2016, the International Society for the Study of Vulvovaginal Disease (ISSVD), developed treatment recommendations by species of yeast. Interestingly, the only non-compounded azole that covers the top 5 most prevalent species of yeast (C. albicans, C. glabrata, C. parapsilosis, C. krusei and S. cerevisiae), 
is miconazole. Thus, miconazole is the society's only recommended antifungal azole treatment for C. glabrata, the most prevalent non-albicans yeast species.

Because of the different methods by which patients can receive VVC treatment, (oral by prescription following contact with HCP or by phone; or OTC with/without interaction or notification with a HCP), providers miss feedback from patients on the success and satisfaction of their treatment as this interaction is bypassed by direct OTC purchase or by telephone prescribing/recommendation. Although miconazole has been shown to be highly effective in clinical and laboratory studies, it is just as important to understand the patient perspective and a level of satisfaction with over-the-counter products, as they may have been used prior to patients visit or recommended by the HCP.

\section{Objectives}

A Trial Program was initiated among patients, nurses and physicians to assess patients' experience and satisfaction with topical miconazole and HCP reasons for dispensing topical miconazole or tioconazole. The objectives of the study were to:

1) Provide hands on experience with dosages and forms.

2) Give HCP's experience with selecting the best topical OTC product for the specific history or symptoms.

3) Record data from patients and gain insights and feedback about the success and safety of topical miconazole/tioconazole in patients with first time or recurrent VVC.

\section{Methods}

Physicians and advanced practice professionals were given 3 samples of each of the following miconazole treatment options to dispense to their gynecologic patients at least age 18 or greater for the treatment of yeast infections:

1) tioconazole ointment $6.5 \%$ (Monistat ${ }^{\circledR} 1$ Simple Therapy ${ }^{\mathrm{TM}}$ )

2) miconazole ovule $1200 \mathrm{mg}$. (Monistat ${ }^{\circledR} 1$ Complete Therapy)

3) miconazole ovule $200 \mathrm{mg} \times 3$ (Monistat ${ }^{\circledR} 3$ Complete Therapy)

4) miconazole cream $100 \mathrm{mg} \times 7$ (Monistat ${ }^{\circledR} 7$ Complete Therapy)

5) In addition to the intra-vaginal treatment, Monistat "complete" products include a tube of miconazole cream for external use and cleansing wipes (Coolwipes ${ }^{\circledR}$ ).

Vulvovaginal candidiasis was diagnosed clinically if signs of vulvar or vaginal erythema with thick white discharge were noted and symptoms of vulvar or vaginal itching and/or irritation were noted. Microbiologic confirmation was often performed but not required and not reported.

Both HCPs and patients were asked to fill out a form. HCPs, including physicians (OBGYN), nurse practitioners (NP), certified nurse midwifes (CNM) and physician assistants (PA) were asked to provide, the name of the dispensed product and the reasons for dispensing the specific product. 
Only one course of treatment was provided, and the duration matched the specific product given of 1,3 , or 7 consecutive days.

Patients were asked to report on their overall experience and satisfaction with the topical products by responding to the following questions and statements, as shown in the Table 1 below.

As no identifying information was collected, the study was deemed exempt from the Institutional Research Review Board.

\section{Results}

\section{1) HCP Respondents}

The Trial Program was conducted from June to October 2015. A total of 2961 NP/CNM/PA and 3007 OB/GYN physicians enrolled in the Trial Program for a total of 5968, with over 3086 reporting on patients (Figure 1).

Participants were selected from 11 states that had the highest concentration of $\mathrm{OB} / \mathrm{GYN}$ healthcare professionals.

Aside from confirming reaching the age of 18 and state of origin, no other identifying information was collected as the study operated as an exempt protocol.

As shown in Figure 1, of the HCPs who enrolled, 52\% (3086) responded. Of these, 60\% (1821) were NPs, CNMs and PAs and 40\% (1265) were OB/GYN physicians.

\section{2) Patient Respondents}

Responses were received from 317 patients; each patient received one product and one card. The number of patient reports from HCPs totaled 19,386 (12,600 from NP/CNM/PA and 6786 from OB/GYN physicians). About a third of patients were first-time yeast sufferers (Figure 2).

3) Patient Findings

An analysis of the responses revealed that almost all patients reported fast symptom relief with any topical miconazole/tioconazole treatment (Figure 3).

Table 1. Questions and possible responses.

\begin{tabular}{|c|c|}
\hline Question & Possible Responses \\
\hline Was this your first yeast infection? & (Yes/No) \\
\hline $\begin{array}{l}\text { Relieved my symptoms (itching, burning) } \\
\text { quickly }\end{array}$ & (Yes/No) \\
\hline Cured my yeast infection within 7 days & (Yes/No/Don't know) \\
\hline $\begin{array}{c}\text { Did you call the doctor back for reasons } \\
\text { related to your treatment? }\end{array}$ & (Yes/No) \\
\hline Would use again, if needed & (Yes/No/Don't know) \\
\hline Overall satisfaction & (not satisfied 12345 very satisfied) \\
\hline Satisfaction with external cream? & (not satisfied 12345 very satisfied N/A-did not use) \\
\hline Satisfaction with cleansing wipes & (not satisfied 12345 very satisfied N/A-did not use) \\
\hline
\end{tabular}




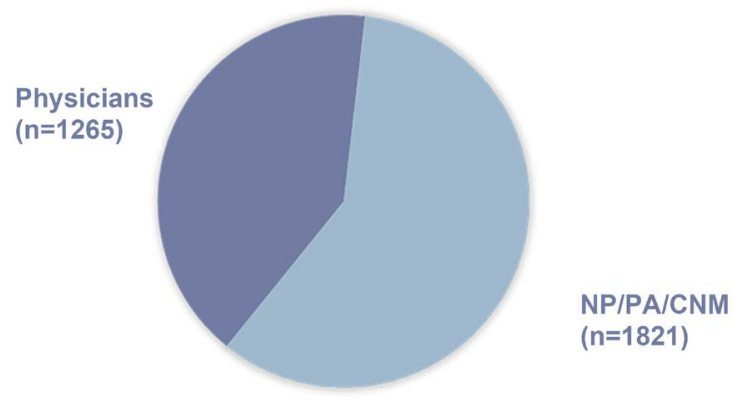

Figure 1. HCP respondents $(\mathrm{n}=3086)$.

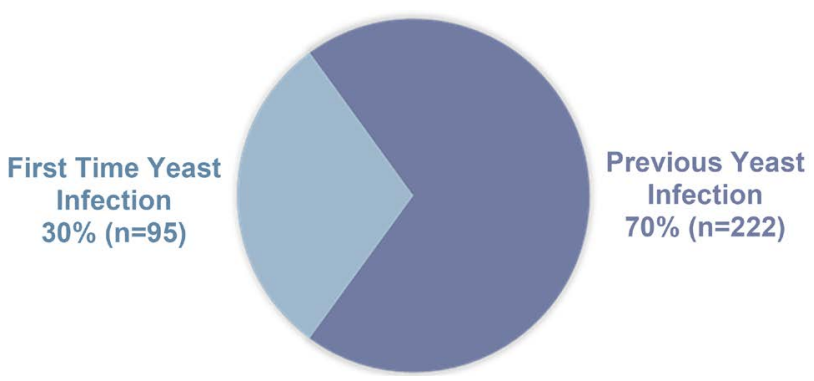

Figure 2. First time or previous yeast infections.

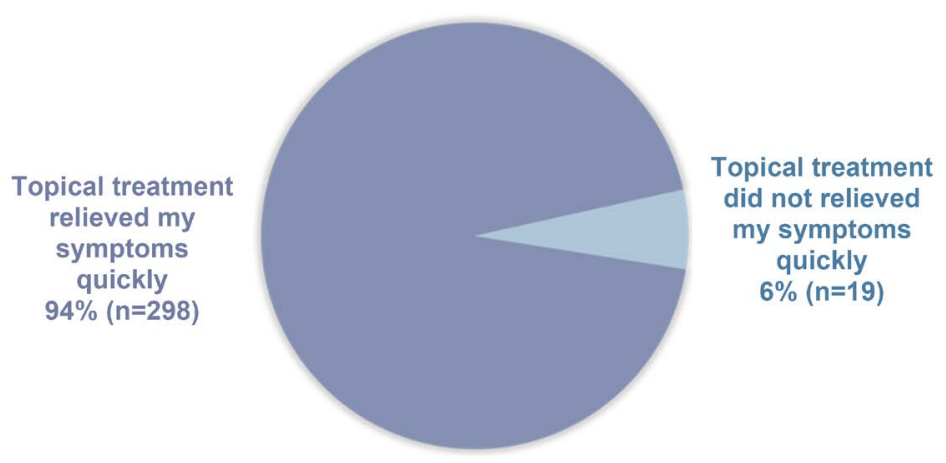

Figure 3. Symptom relief with topical miconazole/tioconazole $(\mathrm{n}=317)$.

When evaluating the individual treatment options, the perception of benefit was associated with specific treatment options (Table 2).

As mentioned previously, almost all patients reported that topical miconazole/tioconazole cured their infection within seven days (Figure 4).

Moreover, almost all patients would use miconazole/tioconazole again (Figure 5).

As all agents were topical, side effects were minimal (less than 1\%) and local, with vulvar burning the predominant symptom. For the $3 \%$ of patient respondents who would not use or were not sure they would use topical miconazole again, the primary reasons were that they did not feel quick symptom relief, did not have had their infection cured within 7 days, or experienced "allergic reaction" and burning sensation after use.

Healthcare professionals recommended the various miconazole/tioconazole products for a variety of reasons that are listed in Table 2 . 
Table 2. Healthcare professional reasons for recommending (miconazole 1-day $=\mathrm{M} 1$, miconazole 3-day $=$ M3, miconazole 7-day $=$ M7, tioconazole 1 -day = Tio1).

\begin{tabular}{|c|c|}
\hline Benefit & Strongest Product Associations and \# of Responses \\
\hline Miconazole efficacy & $\begin{array}{c}\text { M1: } 983 \text { (41\%), M3: } 1994 \text { (41\%), M7: } 2940 \text { (46\%) } \\
\text { Total: } 5917(43 \%)\end{array}$ \\
\hline $4 \mathrm{x}$ faster relief & $\begin{array}{c}\text { M1: } 761 \text { (32\%), M3: } 1545(31 \%), \text { M7: } 1641(26 \%) \\
\text { Tio1: } 2556(45 \%) \\
\text { Total: } 6503(34 \%)\end{array}$ \\
\hline Safer vs. oral $\mathrm{Rx}$ & $\begin{array}{c}\text { M1: } 324(13 \%), \text { M3: } 1145(23 \%), \text { M7: } 1721(27 \%) \text {, } \\
\text { Tio1: } 1631(29 \%) \\
\text { Total: } 4821(25 \%)\end{array}$ \\
\hline Patient prefers less mess ovule & M1: 1049 (44\%), M3: 1276 (26\%) Total: 2325 (32\%) \\
\hline $\begin{array}{l}\text { Women on OCs \& oral } \\
\text { anti-diabetics }+ \text { other drugs }\end{array}$ & $\begin{array}{c}\text { M1: } 450 \text { (19\%), M3: } 851(17 \%), \text { M7: } 617 \text { (10\%), } \\
\text { Tio1: } 1467(26 \%) \\
\text { Total: } 3385(18 \%)\end{array}$ \\
\hline $\begin{array}{l}\text { Resistance of oral fluconazole } \\
\text { increasing }\end{array}$ & $\begin{array}{c}\text { M1: } 380(16 \%), \text { M3: } 654(13 \%), \text { M7: } 681(11 \%), \\
\text { Tio1: } 797(14 \%) \\
\text { Total: } 2512(13 \%)\end{array}$ \\
\hline Patient failed on fluconazole & $\begin{array}{c}\text { M1: } 477(20 \%), \text { M3: } 507(10 \%), \text { M7: } 828(13 \%) \\
\text { Tio1: } 728(13 \%) \\
\text { Total: } 2540(13 \%)\end{array}$ \\
\hline Preferred longer duration & M7: $3618(57 \%)$ \\
\hline Safe in pregnancy & M7: 3025 (47\%) \\
\hline
\end{tabular}

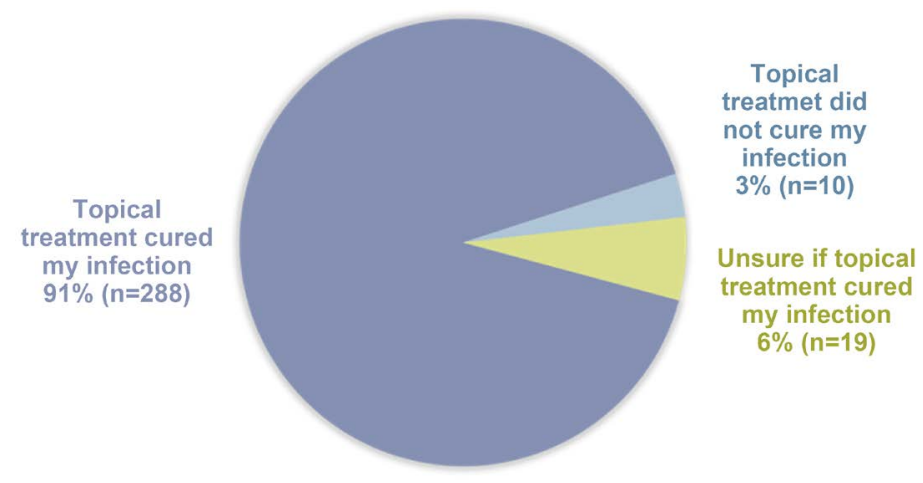

Figure 4. Treatment cure within 7 days.

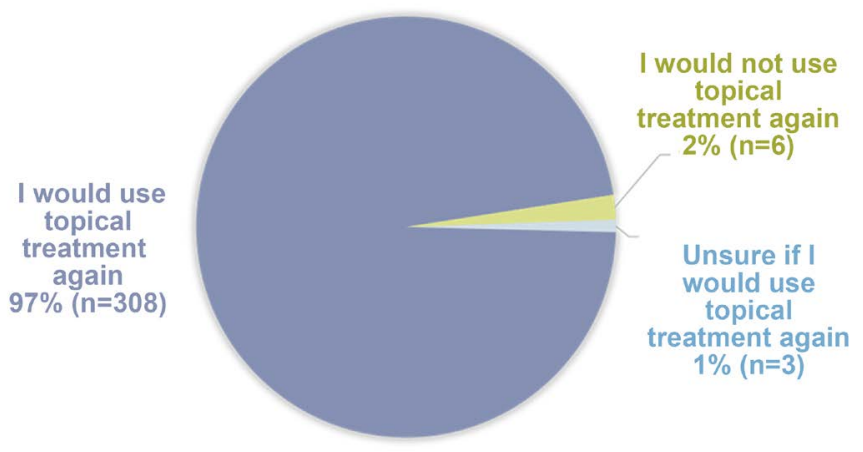

Figure 5. Intended future use of topical miconazole/tioconazole. 
Total miconazole/tioconazole dispensed 19,386, Total miconazole dispensed 13,697, Total ovules (M1, M3) dispensed 7309.

\section{Discussion}

Vulvovaginal candidiasis is one of the most common gynecologic disorders in women today. While in the past, most treatments required a prescription, effective over-the-counter agents have been available for more than a generation. Therefore, supervision and oversight of candidiasis diagnosis and treatment by licensed medical practioners is not commonly occurring. During this time, the question arises; does the patient who presents to a medical office today represent the same patient previously seen a decade ago? Are they first time sufferers? We assume that patients with recurrent infections are familiar with their symptoms and treatment options, many of which are over-the-counter, and therefore bypassing the medical office? Are most patients who self-medicate with over-the-counter anti-fungal agents successful and satisfied with their results, or are they failures either due to incorrect self-diagnosis or inadequate treatment choices or selection?

From these results it appears that most (2/3) are not first time sufferers, but have had previous infections. When the patients in this trial were treated with over-the-counter topical miconazole, $94 \%$ achieved symptom relief and most (91\%) believed their infection was cured. Therefore, the great majority were successfully treated with the miconazole/tioconazole, despite having had a previous infection. It is often assumed that patients with recurrent infections are more difficult to treat, since these patients may have failed OTC agents and are now seeking in-office care.

There has been a growing trend for providers to write for a prescription agent believing it is more effective. However, these data demonstrate that oral miconazole/tioconazole therapy is highly effective clinically in both primary or repeat infections. Also, it may be that it is actually these oral agents that are experiencing repeated failures, giving the impression of recalcitrant infections. Overuse, or misuse of oral agents are also clinically more important now, as adverse effects, especially in pregnancy, have been described with systemic oral agents. This has recently been reflected in changes to the CDC and ISVDD recommendations, which suggest utilizing topical agents, such as miconazole, for infections suspected to be caused by non-albicans Candida and in pregnant women.

While the strengths of the study are related to the large number of health care providers who responded and a thorough examination of their dispensing/prescribing habits, the study is limited by the subjective nature of the patients' responses. Also, objective, microbiologic confirmation of candida clearance would have been helpful.

\section{Conclusions}

The Patient Trial program provided feedback from thousands of prescribers and 
many patients including attitudinal information about the symptom relief and rationale for choosing specific products based on dosages and forms.

Some of the key findings from this study are listed below:

1) Nearly all patients were satisfied with miconazole (95\%) and would use miconazole again (97\%), showing that patients will accept a recommendation of an over-the-counter drug from health professionals.

2) $100 \%$ of first time sufferers would use miconazole again.

3) Very few patients, 3\%, were dissatisfied with miconazole.

4) Almost all reported their yeast infection was cured (91\%) and experienced quick symptom relief (94\%).

5) Patients were also satisfied with the external cream (93\%) and cleansing wipes (96\%) which provided additional soothing and symptom relief.

Therefore, assumptions that topical over the counter antifungal are ineffective in patients with either primary or recurrent infections are incorrect. Care must be taken not to assume that oral agents are necessary, with the excellent results and satisfaction with the topical agents, our recommendation is that prescription of oral agents should be used with caution until studies are completed proving equal efficiency and safety.

\section{Conflicts of Interest}

The authors declare no conflicts of interest regarding the publication of this paper.

\section{References}

[1] Mintz, J.D. and Martens, M.G. (2013) Prevalence of Non-Albicans Candida Infections in Women with Recurrent Vulvovaginal Symptomatology. Advances in Infectious Diseases, 3, 238-242. https://doi.org/10.4236/aid.2013.34035

[2] Centers for Disease Control and Prevention, Sexually Transmitted Diseases Treatment Guidelines (2015) http://www.cdc.gov/std/tg2015/tg-2015-print.pdf

[3] Sobel, J.D., Wiesenfeld, H.C., Martens, M.G., et al. (2004) Maintenance Fluconazole Therapy for Recurrent Vulvovaginal Candidiasis. The New England Journal of Medicine, 351, 876-883. https://doi.org/10.1056/NEJMoa033114

[4] van Heusden, A.M., Merkus, H.M., Corbeij, H.S., et al. (1990) Single-Dose Oral Fluconazole versus Single-Dose Topical Miconazole for the Treatment of Acute Vulvovaginal Candidosis. Acta Obstetricia et Gynecologica Scandinavica, 69, 417-422. https://doi.org/10.3109/00016349009013305

[5] Bruggemann, R.J., Alffenaar, J.W., Blijlevens, N.M., et al. (2009) Clinical Relevance of the Pharmacokinetic Interactions of Azole Antifungal Drugs with Other Coadministered Agents. Clinical Infectious Diseases, 49, 1441-1458. https://doi.org/10.1086/598327

[6] Sinofsky, F.E. and Pasquale, S.A. (1998) The Effect of Fluconazole on Circulating Ethinyl Estradiol Levels in Women Taking Oral Contraceptives. American Journal of Obstetrics \& Gynecology, 178, 300-304. https://doi.org/10.1016/S0002-9378(98)80016-1

[7] Willams, N.S., Phillips, N. and Bachmann, G. (2015) Oral Compared with Local 
Antifungal Treatment of Yeast Infection: Comparison of Time to Symptomatic Relief. Obstetrics \& Gynecology, 125, 11S.

https://doi.org/10.1097/01.AOG.0000463549.01588.2b

[8] Marchaim, D., Lemanek, L., Bheemreddy, S., Kaye, K.S. and Sobel, J.D. (2012) Fluconazole-Resistent Candida albicans Vulvovaginitis. Obstetrics and Gynecology, 120, 1407-1414. https://doi.org/10.1097/AOG.0b013e31827307b2

[9] Shabid, Z. and Sobel, J.D. (2009) Reduced Fluconazole Susceptibility of Candida albicans Isolates in Women with Recurrent Vulvovaginal Candidiasis: Effects of Long-Term Fluconazole Therapy. Diagnostic Microbiology and Infectious Disease, 64, 354-356. https://doi.org/10.1016/j.diagmicrobio.2009.03.021

[10] Vazquez, J.A., Peng, G., Sobel, J.D., et al. (2001) Evolution of Antifungal Susceptibility among Candida Species Isolates Recovered from Human Immunodeficiency Virus-Infected Women Receiving Fluconazole Prophylaxis. Clinical Infectious Diseases, 33, 1069-1075. https://doi.org/10.1086/322641 